\title{
BAND STRUCTURE OF DILUTE METASTABLE Co-Ag ALLOYS
}

\author{
J.A. MORKowsKi* AND A. SZAJEK \\ Institute of Molecular Physics, Polish Academy of Sciences \\ M. Smoluchowskiego 17, 60-179 Poznan, Poland
}

\begin{abstract}
Co-Ag systems have recently attracted considerable attention in application oriented research of ferromagnetic thin films and multilayers. Due to low miscibility of $\mathrm{Ag}$ in $\mathrm{Co}$ it is easy to fabricate thin $\mathrm{Co}-\mathrm{Ag}$ films of granular structure, promising as a high magnetoresistance material. It was also shown that using modern technology it is possible to deposit thin films of Co-Ag forming metastable homogeneous alloys for compositions far exceeding the solubility limit. In the present paper results of electronic structure calculations by the tight-binding linear muffin-tin orbital method are reported. Computations were done for hypothetical uniform ordered $\mathrm{Co}_{100-x} \mathrm{Ag}_{x}$ alloys assuming proper extended unit cells to simulate concentrations of $x=25,12.5,6.25,3.125$ at.\%. The results were used to calculate the photoemission spectra. The calculated photoemission spectra are in fair agreement with experimental data supporting the conclusion of existence of a homogeneous though metastable alloy in the dilute limit. On the contrary, superposition of pure Co and Ag spectra implied for precipitate of $\mathrm{Ag}$ in Co cannot explain the experimental data.
\end{abstract}

PACS numbers: 71.20.-b, 82.80.Pv

\section{Introduction}

Since the recent discovery of giant magnetoresistance (GMR) and establishing the spin-dependent scattering of the conduction electrons as a mechanism responsible for GMR, there has been growing interest in heterogeneous materials like films separated by nonmagnetic spacers, granular systems made of magnetic grains embedded in nonmagnetic matrices or granular multilayer systems. In choosing the best combinations of ferromagnetic metal (or alloy) with nonmagnetic spacer or matrix, low relative miscibility is a criterion. The Co-Ag system is particularly convenient and widely studied since homogeneous alloys $\mathrm{Co}_{100-c} \mathrm{Ag}_{c}$ are stable only for $c \leq 1$ at\%. The granular Co-Ag multilayer systems are believed to have a great potential for applications based upon the GMR effect. Thus it is important

*corresponding author; e-mail: jmorkows@ifmpan.poznan.pl 
to know the conditions for alloying versus separation of constituents for developing proper technological procedures to get optimal multilayers.

In a recent study $[1,2]$ of the interlayer exchange coupling of Co layers separated by a nonmagnetic spacer layer of $\mathrm{Ru}$ it was observed that an addition of small amounts of $\mathrm{Ag}$ to $\mathrm{Co}$ in the magnetic layer resulted in large changes of the phases of the oscillating interlayer exchange coupling, while the oscillation periods remained only slightly modified. The controlled process of deposition of the layer system and the characterisation of the trilayer system obtained justify the assumption that the $\mathrm{Ag}$ doped Co layers were in fact $\mathrm{Co}-\mathrm{Ag}$ alloys for $\mathrm{Ag}$ concentrations up to 8 at\% (possible $\mathrm{Ag}$ clusters could only have diameters not exceeding $10 \AA[2])$. The Co- $\mathrm{Ag}$ alloys for concentrations exceeding the solubility limit are metastable systems formed by epitaxial grows of layers at low temperatures of the substrate to suppress migration of $\mathrm{Ag}$, so preventing the clustering of $\mathrm{Ag}$ atoms. The dependence of the phase of the interplanar exchange interaction appeared consistent with electronic band structure data, supporting the notion of alloying $\mathrm{Ag}$ in Co beyond the solubility limit.

The existence of $\mathrm{CoAg}$ alloys for concentrations of $\mathrm{Ag}$ up to 6 at\% was soon after confirmed [3]. Thin films of $\mathrm{Co}-\mathrm{Ag}$ and also $\mathrm{Co}-\mathrm{Au}$ were fabricated by co-evaporation onto a cold $\mathrm{Si}(111)$ substrate. High-resolution $\mathrm{X}$-ray photoemission spectroscopy (XPS) was used to study the structure of the valence band of the Co-noble metal systems. It was concluded [3] that the XPS spectra (for Ag concentration less than 6 at\%) cannot be explained by superposition of properly weighted spectra for pure Co and $\mathrm{Ag}$, thus ruling out extensive clustering. On the contrary, the results suggested that $\mathrm{Co}$ and $\mathrm{Ag}$ form a metastable dilute alloy. The behaviour of the XPS spectra [3] was consistent with the reported data [2] on the band structure of dilute $\mathrm{Co}-\mathrm{Ag}$ alloys. In the present paper the detailed results of the calculations of the electronic band structure for dilute ordered $\mathrm{Co}-\mathrm{Ag}$ alloys are reported and used to calculate the theoretical XPS spectra. Included are also some data for Co-M $(\mathrm{M}=\mathrm{Au}, \mathrm{Cu}, \mathrm{Ru})$.

\section{Electronic band structure}

The electronic structure and magnetic moments were calculated based on the spin-polarized tight-binding linear muffin-tin orbital (TB LMTO) method in the atomic sphere approximation (ASA) $[4,5]$. In the ASA the unit cell is filled by Wigner-Seitz spheres having the same total volume

$$
\frac{4}{3} \pi \sum_{j=1}^{N} S_{j}^{3}=N \frac{4}{3} \pi S_{\mathrm{av}}^{3}=V,
$$

where $j$ is the index of an atom in the unit cell, $N$ is the number of atoms in the cell, $S_{j}$ is the Wigner-Seitz radius of the $j$-th atom, $S_{a v}$ is the average Wigner-Seitz radius, $V$ is the volume of the unit cell. In our case the unit cells contain maximum 32 atoms. The overlap volume of the muffin-tin spheres is about $7.9 \%$. The standard combined corrections [4] for overlapping were used to compensate for errors due to the ASA. The spin-orbit interactions were taken into account in the form 
proposed by Min and Jang [6]. The exchange correlation potential was assumed in the form proposed by Perdew et al. [7] with nonlocal corrections. The starting atomic configurations were taken as: core $(\mathrm{Kr})+4 d^{10} 5 s^{1}$ for $\mathrm{Ag}$ and core(Ar) + $3 d^{7} 4 s^{2}$ for Co atom. The experimental values of the lattice constants were used in the calculations. For the f.c.c. system the lattice constant of a f.c.c. Cu substrate was taken [8] (3.615 $\AA$ ) and for the h.c.p. system the h.c.p. Co lattice constants were taken [9] equal to $a=2.5074 \AA$ and $c=4.0699 \AA$. Based on these values the supercells were constructed. The self-consistent calculations were performed for about $720 k$-points in the irreducible wedge of the Brillouin zone, depending on the symmetry of a system. The tetrahedron method [10-12] was used for integration over the Brillouin zone. The iterations were repeated until the energy eigenvalues of the consecutive iteration steps were the same within an error of $0.01 \mathrm{mRy}$.

The electronic band structure was calculated for hypothetical ordered dilute $\mathrm{Co}-\mathrm{Ag}$ alloys for a few concentrations as $25,12.5,6.25$, and 3.125 at\% of $\mathrm{Ag}$, which can be simulated by the choice of supercells as depicted in Fig. 1. The Co-Ag layers in the experiments [2] were grown perpendicularly to the [111] direction. In pure Co layers a high concentration of stacking faults was observed [2] indicating a mixture of f.c.c. and h.c.p. structures. Therefore band structure calculations were done for both h.c.p. and f.c.c. structures.
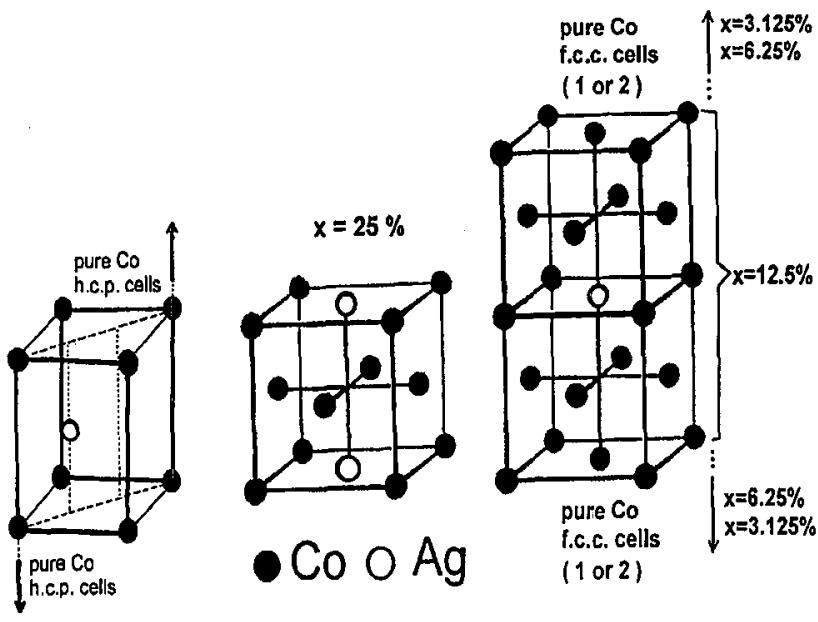

Fig. 1. The h.c.p. and f.c.c. unit cells used in the calculations for the $\mathrm{Co}_{1-x} \mathrm{Ag}_{x}$ systems.

In Fig. 2 the densities of states for spins up and down are presented for h.c.p. Co-Ag alloys for $\mathrm{Ag}$ concentrations of $0,6.25,12.5$, and 25 at\%, respectively. Similar data for f.c.c. structure and an extended concentration range are provided in Fig. 3. With an increasing concentration of $\mathrm{Ag}$ the contribution to the total DOS from the $4 d$ band of $\mathrm{Ag}$, centred around $-6 \mathrm{eV}$, is more and more pronounced.

For the purpose of comparison with the other systems studied [2] the total DOS (summed for both spin directions) for f.c.c. $\mathrm{Co}_{0.75} \mathrm{M}_{0.25}$ systems ( $\mathrm{M}=\mathrm{Au}$, $\mathrm{Cu}, \mathrm{Ru}$ ) are given in Fig. 4 . 

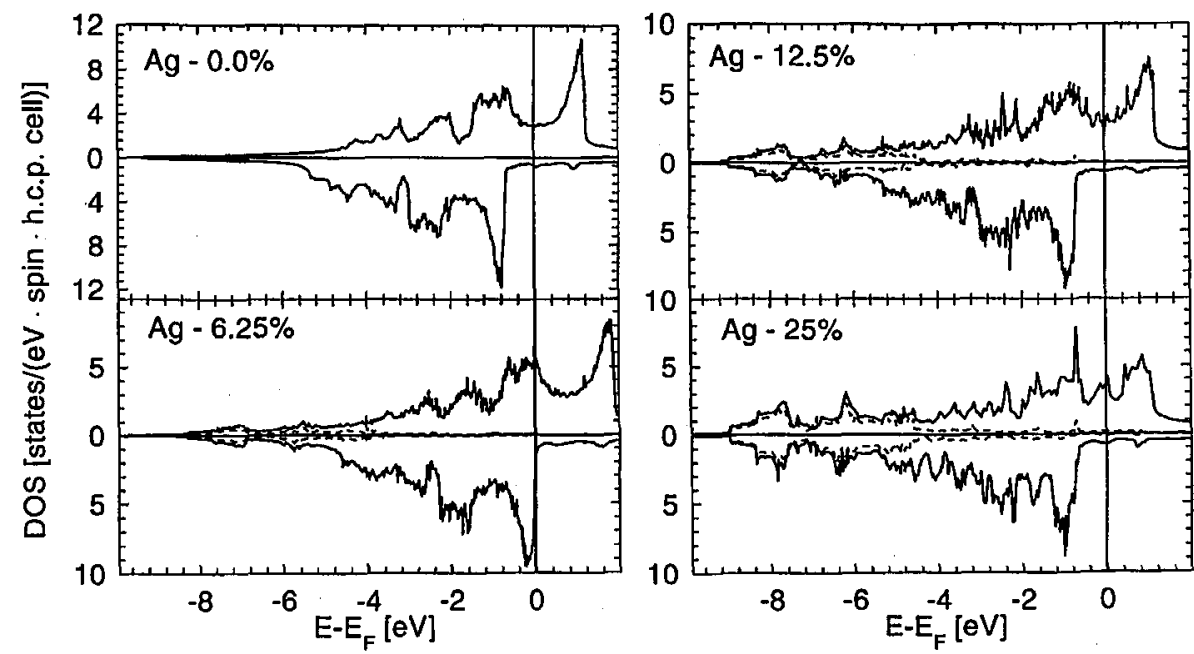

Fig. 2. The spin resolved DOS [states $/(\mathrm{eV} \cdot \operatorname{spin} \cdot$ unit cell $)$ for the h.c.p. $\mathrm{Co}_{1-x} \mathrm{Ag}_{x}$ alloys.
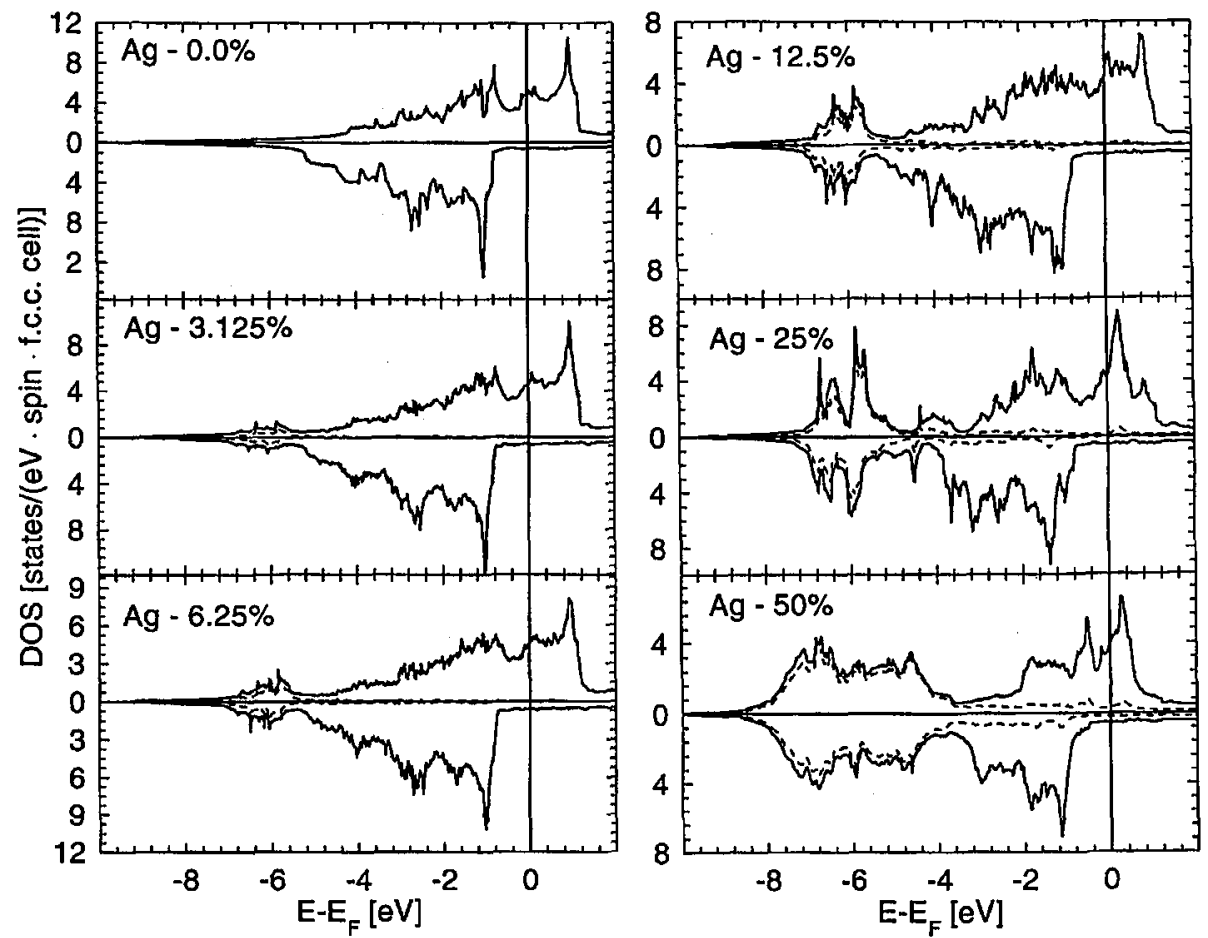

Fig. 3. The spin resolved DOS for the f.c.c. $\mathrm{Co}_{1-x} \mathrm{Ag}_{x}$ systems. 


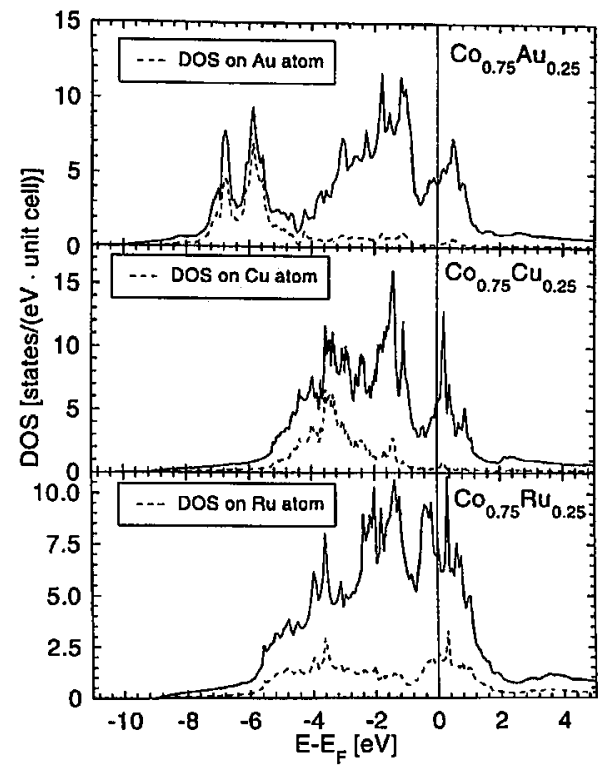

Fig. 4. The total DOS for the f.c.c. $C_{0.75} M_{0.25}$ systems $(M=A u, C u, R u)$.

TABLE I

Calculated magnetic moments (in Bohr magnetons) at Co and $\mathrm{Ag}$ atoms for f.c.c. Co-Ag alloys. Numbers in parentheses in the first column specify the positions of the respective atoms.

\begin{tabular}{c|c|c|c|c|c}
\hline \hline Magn. moments $\left[\mu_{\mathrm{B}}\right]$ & $3.125 \%$ & $6.25 \%$ & $12.5 \%$ & $25 \%$ & $0 \%$ \\
\hline $\mathrm{Ag}(1 / 2,1 / 2,0)$ & 0.030 & 0.027 & 0.027 & -0.047 & - \\
$\mathrm{Co}(000)$ & 1.644 & 1.638 & 1.644 & 1.675 & 1.718 \\
$(1 / 2,0,1 / 2)$ & 1.693 & 1.687 & 1.699 & 1.675 & - \\
$(1 / 2,1 / 2,1)$ & 1.724 & 1.718 & 1.723 & - & - \\
$(001)$ & 1.741 & 1.738 & 1.754 & - & - \\
$(1 / 2,0,3 / 2)$ & 1.738 & 1.737 & - & - & \\
$(1 / 2,1 / 2,2)$ & 1.724 & 1.716 & - & - & - \\
$(002)$ & 1.734 & 1.734 & - & - & - \\
$(1 / 2,0,5 / 2)$ & 1.733 & - & - & - & - \\
$(1 / 2,1 / 2,3)$ & 1.729 & - & - & - & - \\
$(003)$ & 1.733 & - & - & - & - \\
$(1 / 2,0,7 / 2)$ & 1.733 & - & - & - & - \\
$(1 / 2,1 / 2,4)$ & 1.732 & - & - & - & - \\
$(004)$ & 1.732 & - & - & - & -
\end{tabular}


TABLE II

Magnetic moments for h.c.p. alloys. Numbers in the first column enumerate the planes in the unit cell.

\begin{tabular}{c|c|c|c|c|c}
\hline \hline Magnetic & $3.125 \%$ & $6.25 \%$ & $12.5 \%$ & $25 \%$ & $0 \%$ \\
moments $\left[\mu_{\mathrm{B}}\right]$ & & & & & \\
\hline $\mathrm{Co}(15)$ & 1.6882 & - & - & - & - \\
$\mathrm{Co}(14)$ & 1.6882 & - & - & - & - \\
$\mathrm{Co}(13)$ & 1.6889 & - & - & - & - \\
$\mathrm{Co}(12)$ & 1.6885 & - & - & - & - \\
$\mathrm{Co}(11)$ & 1.6886 & - & - & - & - \\
$\mathrm{Co}(10)$ & 1.6878 & - & - & - & - \\
$\mathrm{Co}(9)$ & 1.6877 & - & - & - & - \\
$\mathrm{Co}(8)$ & 1.6894 & - & - & - & - \\
$\mathrm{Co}(7)$ & 1.6886 & 1.6625 & - & - & - \\
$\mathrm{Co}(6)$ & 1.6900 & 1.6632 & - & - & - \\
$\mathrm{Co}(5)$ & 1.6858 & 1.6600 & - & - & - \\
$\mathrm{Co}(4)$ & 1.6874 & 1.6641 & - & - & - \\
$\mathrm{Co}(3)$ & 1.6809 & 1.6562 & 1.6493 & - & - \\
$\mathrm{Co}(2)$ & 1.7107 & 1.6961 & 1.6820 & - & - \\
$\mathrm{Co}(1)$ & 1.5805 & 1.5918 & 1.5700 & 1.5490 & 1.6446 \\
$\mathrm{Ag}(0)$ & 0.0001 & 0.0076 & 0.0049 & 0.0017 & - \\
$\mathrm{Co}(1)$ & 1.5802 & 1.5918 & 1.5700 & 1.5490 & - \\
$\mathrm{Co}(2)$ & 1.7108 & 1.6961 & 1.6820 & 1.7204 & - \\
$\mathrm{Co}(3)$ & 1.6808 & 1.6562 & 1.6364 & - & - \\
$\mathrm{Co}(4)$ & 1.6880 & 1.6641 & 1.6364 & - & - \\
$\mathrm{Co}(5)$ & 1.6857 & 1.6600 & - & - & - \\
$\mathrm{Co}(6)$ & 1.6889 & 1.6632 & - & - & - \\
$\mathrm{Co}(7)$ & 1.6886 & 1.6613 & - & - & - \\
$\mathrm{Co}(8)$ & 1.6895 & 1.6613 & - & - & - \\
$\mathrm{Co}(9)$ & 1.6877 & - & - & - & - \\
$\mathrm{Co}(10)$ & 1.6876 & - & - & - & - \\
$\mathrm{Co}(11)$ & 1.6885 & - & - & - & - \\
$\mathrm{Co}(12)$ & 1.6884 & - & - & - & - \\
$\mathrm{Co}(13)$ & 1.6887 & - & - & - & - \\
$\mathrm{Co}(14)$ & 1.6880 & - & - & - & - \\
$\mathrm{Co}(15)$ & 1.6884 & - & - & - & - \\
$\mathrm{Co}(16)$ & 1.6878 & - & - & & \\
& & & & & - \\
\hline
\end{tabular}


The calculated magnetic moments on Co atoms in various positions within the unit cell, as defined in Fig. 1, are summarized in Tables I and II, for f.c.c. and h.c.p. phases, respectively. The experimental values for Co are $1.739 \mu_{\mathrm{B}}$ for f.c.c. [13] and $1.715 \mu_{\mathrm{B}}$ for h.c.p. [14].

\section{XPS spectra}

To probe the occupied part of the electronic band structure the photoemission spectra were calculated. The XPS spectrum was calculated from the partial densities of states DOS(E) weighted with atomic photoemission cross-sections [15] for the photon energy equal to $1486.6 \mathrm{eV}$ ( $\mathrm{Al} K_{\alpha}$ source). The finite experimental resolution was taken into account in the calculations by convoluting the weighted DOS(E) with an energy dependent Lorentzian function with a half-width $\delta=0.3 \mathrm{eV}$.

The calculated XPS spectra for h.c.p. and f.c.c. Co-Ag alloys are presented in Figs. 5 and 6 , respectively. For f.c.c. alloys (Fig. 6) the weighted by concentration XPS spectra $J_{\text {clusters }}=(1-x) J_{\mathrm{Co}}+x J_{\mathrm{Ag}}$ are also provided. They are relevant for heterogeneous systems consisting of clusters of the one component in a matrix of the other. For a concentration of 3.125 at\% the calculated XPS intensity for the alloy, $J_{\text {alloy }}$, is compared with $J_{\text {clusters }}$ and with the experimental intensity for a close concentration of 2.6 at\%, taken from [3]. It is evident that the characteristic features of $J_{\text {alloy }}$ agree with those from experiment, whereas $J_{\text {clusters }}$ is distinctively different. Similar agreement is observed for a concentration of 6 at\% of Ag.

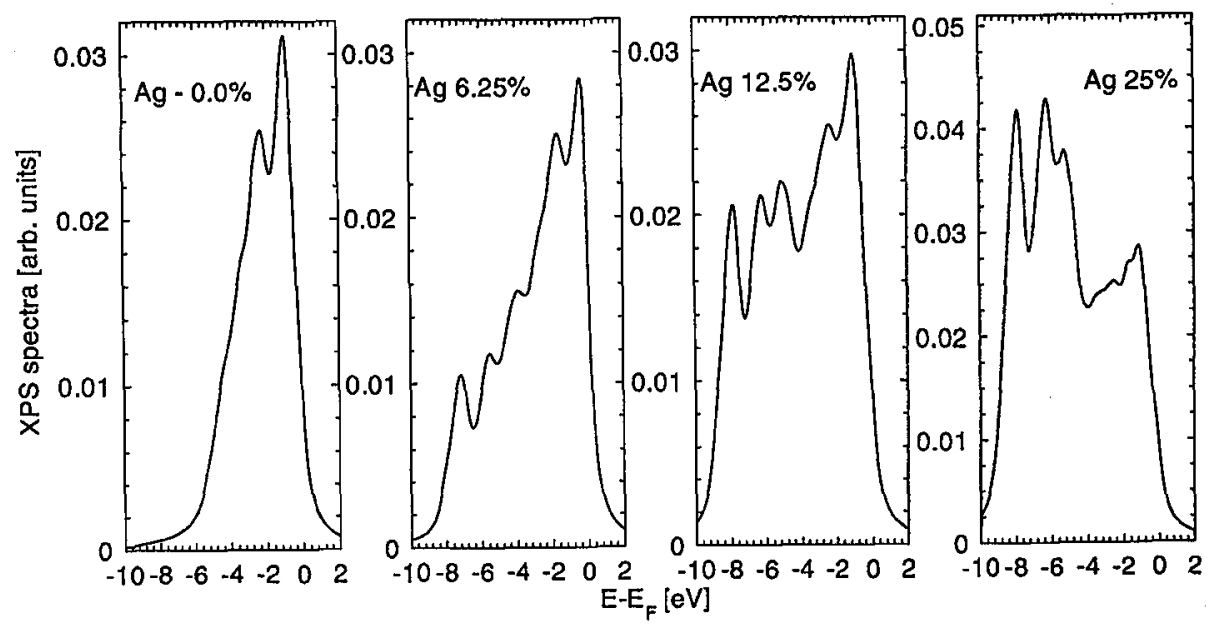

Fig. 5. The XPS spectra for the h.c.p. $\mathrm{Co}_{1-x} \mathrm{Ag}_{x}$ alloys. 

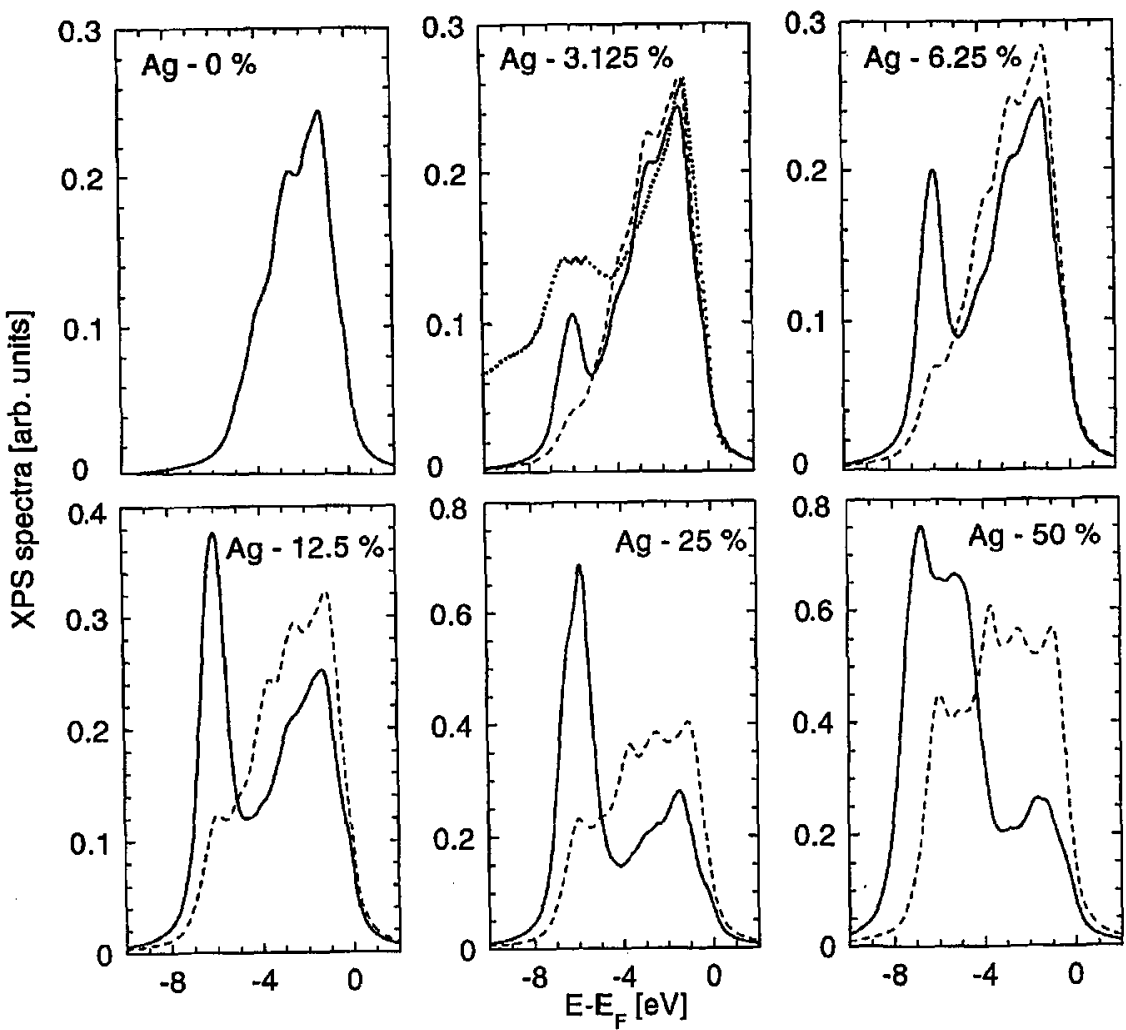

Fig. 6. The XPS spectra for the f.c.c. $\mathrm{Co}_{1-x} \mathrm{Ag}_{x}$ alloys (full lines), compared with weighted superpositions of XPS curves for pure Co and $\mathrm{Ag}$ (broken lines). The panel for $3.125 \% \mathrm{Ag}$ contains also experimental data from [3] (dotted line).

\section{Acknowledgment}

The present research was supported by the Committee for Scientific Research (Poland) grant No. 2P0B 118 14. The band calculations were performed at the Supercomputing and Networking Centre of Poznań.

\section{References}

[1] P. Wigen, Li Zhou, R. Stamps, K. Ounadjela. M. Hehn, J. Gregg, J. Magn. Magn. Mater. 165, 465 (1997).

[2] U. Ebels, R.L. Stamps, L. Zhou, P.E. Wigen, K. Ounadjela, J. Greggs, J. Morkowski, A. Szajek, Phys. Rev. B 58, 6367 (1998).

[3] M. Gester, J.A.D. Matthew, S.M. Thompson, G. Beamson, Phys. Rev. B 59, 15654 (1999).

[4] O.K. Andersen, O. Jepsen, M. Sob, in: Electronic Structure and its Applications, Ed. M.S. Yussouff, Springer, Berlin 1987, p. 2. 
[5] G. Krier, O. Jepsen, A. Burkhardt, O.K. Andersen, The TB-LMTO-ASA Program (source code, version 4.7, a vailable upon request from the program authors).

[6] B.I. Ming, Y.-R. Jang, J. Phys., Condens. Matter 3, 5131 (1991).

[7] J.P. Perdew, J.A. Chevary, S.H. Vosko, K.A. Jackson, M.R. Pederson, D.J. Singh, C. Fiolhais, Phys. Rev. B 46, 6671 (1992).

[8] J.J. de Miguel, A. Cebolada, J.M. Gallego, A. Miranda, C.M. Schneider, P. Schuster, J. Kirschner, J. Magn. Magn. Mater. 93, 1 (1991).

[9] American Institute of Physics Handbook, Ed. P.E. Grey, Mc-Graw-Hill Book Company, New York 1972.

[10] O. Jepsen, O.K. Andersen, Solid State Commun. 9, 1763 (1971).

[11] O. Jepsen, O.K. Andersen, Phys. Rev. B 29, 5965 (1984).

[12] P. Blöchl, O. Jepsen, O.K. Andersen, Phys. Rev. B 49, 16223 (1994).

[13] J. Crangle, Philos. Mag. 46, 499 (1955).

[14] H.P. Meyers, W. Sucksmith, Proc. R. Soc. A 207, 427 (1951).

[15] J.J. Yeh, I. Lindau, At. Data Nucl. Data Tables 32, 1 (1985). 